05

\title{
Особенности тепловых процессов при токовой перегрузке в многослойных ВТСП проводниках
}

\author{
(c) В.А. Мальгинов, ${ }^{1}$ А.В. Мальгинов, ${ }^{1}$ Л.С. Флейшман, ${ }^{2}$ А.С. Ракитин ${ }^{2}$ \\ ${ }^{1}$ Физический институт им. П.Н. Лебедева РАН, \\ 119991 Москва, Россия \\ ${ }^{2}$ ОАО Энергетический институт им. Г.М. Кржижановского, \\ 119071 Москва, Россия \\ e-mail: malginov@sci.lebedev.ru
}

(Поступило в Редакцию 25 октября 2016 г. В окончательной редакции 24 марта 2017 г.)

\begin{abstract}
Детально исследован процесс перехода многослойных ВТСП проводников, предназначенных для использования в электрооборудовании, из сверхпроводящего состояния в резистивное и из резистивного в нормальное при протекании токов, превышающих критический ток. Выявлены условия, способствующие повышению тока теплового срыва многослойных ВТСП ленточных проводников на основе анализа теплового состояния ВТСП ленты в перегруженном режиме, а также приведена оценка максимальной температуры разогрева и ее поперечного градиента во время перехода ВТСП ленты в нормальное состояние с использованием экспериментальных данных по сопротивлению нормальной области и времени восстановления сверхпроводимости.
\end{abstract}

DOI: 10.21883/JTF.2017.10.44995.2077

\section{Введение}

Исследование процессов, происходящих в композитных ВТСП материалах, в особенности в многослойных ленточных проводах, при протекании токов выше критического, имеет значение для создания сверхпроводниковых электротехнических устройств, удовлетворяющих требованиям электрических сетей по надежности, термической и электродинамической стойкости [1].

В настоящее время для ВТСП композитных материалов вопрос о роли факторов, определяющих значение тока теплового перехода в нормальное состояние, еще не имеет однозначного решения. Для криостатической стабилизации сверхпроводящего состояния низкотемпературных композитных сверхпроводников необходимо увеличивать относительную концентрацию нормального металла в них [2]. Однако в [3] для ВТСП ленточных проводов на основании анализа теоретической модели утверждается, что возможность повышения максимально допустимого тока с помощью стабилизирующего покрытия весьма ограничена (до 20\%).

Теоретическое рассмотрение тепловых процессов в ВТСП лентах было выполнено в [3-5]. Однако в этих теоретических моделях все расчеты выполнены для случая, когда охлаждение ВТСП ленты осуществляется хладагентом с постоянным коэффициентом теплоотдачи. В реальности же наличие кризиса кипения жидкого азота (предпочтительного хладагента для ВТСП электрооборудования) играет принципиальную роль для развития тепловой нестабильности в ВТСП лентах. Поскольку строгий теоретический расчет разогрева лент весьма затруднителен и может давать большую погрешность в оценке максимальной температуры, небходимо детальное экспериментальное исследование реальных процессов разогрева, теплоотвода и тех параметров, с помощью которых можно произвести адекватную оценку температуры.
Как было показано в ряде работ [6-8], существует некоторый диапазон токов, превышающих критическое значение, но меньших предельного тока теплового перехода, в котором возможно устойчивое состояние при соблюдении теплового баланса между тепловыделением и теплоотводом. Это так называемое резистивное состояние было теоретически рассмотрено для низкотемпературных сверхпроводников при охлаждении жидким гелием [2]. Однако параметры кривой кипения жидкого гелия приводят к малости диапазона теплового напора (нагрева сверхпроводника) $\Delta T \leq 1 \mathrm{~K}$ [9], что делает наблюдение такого состояния весьма проблематичным. Для ВТСП материалов в жидком азоте в силу достижимого в режиме пузырькового кипения азота значения $\Delta T \leq 20-25 \mathrm{~K}[10]$ устойчивые резистивные состояния уверенно наблюдаются в экспериментах (например, в $[7,8]$ приводятся измеренные значения $\Delta T \leq 8 \mathrm{~K})$.

При превышении током в ВТСП ленте предельного значения тепловой баланс нарушается и происходит значительный нагрев ленты (тепловой „срыв“ или квенч). Ввиду наличия продольной неоднородности ее свойств разогрев имеет локальный характер, что особенно выражено в многослойных ВТСП материалах со сложной структурой $[1,11]$. Неоднородность свойств ВТСП ленточных проводов по длине вместе с низкой скоростью распространения нормальной зоны приводит к локальному перегреву выше $400 \mathrm{~K}$ [1] и необратимой деградации их сверхпроводящих свойств.

Таким образом, для понимания механизмов, способствующих повышению тока теплового срыва, для нахождения причин, вызывающих необратимую потерю сверхпроводимости в ВТСП токонесущих элементах при токовой и тепловой перегрузках, для предотвращения их разрушения при работе в сверхпроводниковых электротехнических устройствах и для создания безынерционного термически стойкого ВТСП токоограничивающего 
устройства [12-16] необходимо детальное исследование процесса перехода как из сверхпроводящего состояния в резистивное, так и из резистивного в нормальное. Этому посвящена настоящая работа.

\section{1. Анализ влияния стабилизирующего покрытия на тепловое состояние ВТСП ленты в условиях токовой перегрузки}

Рассмотрим композитную стабилизированную ВТСП ленту, охлаждаемую жидким азотом (использование других хладагентов в настоящей работе не рассматривается). В выполняемом анализе, имеющем целью рассмотрение протекающих процессов на качественном уровне, под стабилизатором понимается следующее: в ВТСП лентах первого поколения - матрица из хорошо проводящего металла (обычно используется серебро); в лентах второго поколения - слой металла (обычно медный) с гораздо большей электропроводностью, чем у стальной подложки. Изменение сопротивления стабилизирующего слоя в актуальном интервале температур не превышает 20-30\%, что не влияет на получаемые в используемой модели качественные результаты и в расчетах не учитывается. При необходимости такой учет несложно выполнить.

В ВТСП лентах второго поколения по технологическим причинам присутствует защитный слой серебра, однако его толщина $(\sim 1 \mu \mathrm{m})$ на порядок меньше толщины медного стабилизирующего слоя. В силу близости значений удельной электропроводности меди и серебра, в последующем анализе они рассматриваются как единый слой стабилизатора с соответствующим эквивалентным сопротивлением, причем распределение тока между серебром и медью не имеет значения для выполняемого качественного рассмотрения.

ВТСП лента в определенном диапазоне проходящих через нее сверхкритических токов может находиться в стабильном перегруженном режиме [3-8]. Нас будет интересовать стационарное резистивное состояние при постоянном токе, меньшем тока теплового перехода, при котором распределение электрического поля вдоль ленты является однородным $[1,8]$. Учитывая небольшую величину нагрева ленты в таком режиме $[7,8]$, выполним, следуя [3], анализ теплового состояния ленты в нульмерном приближении с использованием модельной степенной вольтамперной характеристики $\left(\mathrm{BAX} E=E\left(I_{s}\right)\right.$, где $I_{s}$ - протекающий в сверхпроводнике постоянный ток, $E-$ напряженность электрического поля) $[3-5,8]$. Полный ток в ленте $I$, задаваемый источником тока, не изменяется при нагреве ленты от температуры хладагента $T_{0}$ до температуры $T$ и складывается из токов в сверхпроводящем слое и стабилизаторе (ток в высокоомной подложке имеет существенно меньшую величину):

$$
I=I_{c}(T)\left[\frac{E}{E_{0}}\right]^{1 / n}+\frac{E l}{R_{n}},
$$

где первое и второе слагаемые описывают ток $I_{s}$ в ВТСП слое и $I_{n}$ в стабилизаторе; $E_{0}-$ значение напряженности электрического поля, используемое в определении критического тока $I_{c}(T)$, линейно убывающего до нуля при нагреве ВТСП до критической температуры $T_{c}$ в соответствии с зависимостью $I_{c}(T)=I_{c}\left(T_{0}\right)\left(T_{c}-T\right) /\left(T_{c}-T_{0}\right) \quad$ (собственное магнитное поле ленты и/или магнитные поля рассеяния в ВТСП электрооборудовании с рабочей температурой $77 \mathrm{~K}$ не превышают $0.1 \mathrm{~T}$ [17] и не оказывают влияния на критические значения тока и температуры); в последующих численных расчетах используется значение $E_{0}=10^{-4} \mathrm{~V} / \mathrm{m}$, при этом конкретное значение $E_{0}$ играет роль только при обезразмеривании уравнений и не влияет на результаты, $n \gg 1-$ показатель степени в модельной $\mathrm{BAX}, l$ - длина ленточного провода; $R_{n}-$ эквивалентное сопротивление стабилизирующего слоя (с учетом его возможного строения из параллельных медного и серебряного слоев).

Напряженность поля, определяемая из (1) при каждом значении температуры и полного тока $E=E(T, I)$, входит в выражение для тепловыделения на единице длины:

$$
Q(T, I)=E(T, I) I
$$

Тепловое состояние ВТСП ленты определяется соотношением между тепловыделением $Q(T, I)$ и теплоотводом в хладагент $W(T)$. Как будет показано ниже, для ВТСП лент со стабилизирующим слоем актуальным механизмом теплоотвода при определении максимального тока теплового срыва является кипение жидкого азота. При этом $W(T)=q_{N 2} P\left(q_{N 2}-\right.$ плотность теплового потока, снимаемого жидкостью с поверхности нагрева, $P$ - периметр поперечного сечения ленты). Как указано в [3], если при повышении температуры кривая тепловыделения $Q(T, I)$ пересекает кривую теплоотвода $W(T)$ сверху, то точка пересечения соответствует устойчивому положению равновесия, в противном случае неустойчивому, сменяющемуся тепловым срывом ВТСП провода в нормальное состояние. Для определения характера пересечения кривых $Q(T, I)$ и $W(T)$ следует сравнить их производные по температуре при различных токах I. Равенство производных достигается при граничном токе между устойчивыми и неустойчивыми состояниями, называемом током теплового перехода или срыва [3]. Таким образом, ток срыва определяется следующими условиями [2]:

$$
Q(T, I)=W(T), \quad \frac{\partial Q(T, I)}{\partial T}=\frac{\partial W(T)}{\partial T} .
$$

Для определения производной тепловыделения $Q(T)$ по температуре после дифференцирования (2) с учетом (1) при условии $I=$ const в результате несложных 
преобразований получаем

$$
\frac{\partial Q}{\partial T}=I \frac{I_{s}}{\left(T_{c}-T\right)}\left[\frac{I_{s}}{n E}+\frac{l}{R_{n}}\right]^{-1} .
$$

В случае отсутствия стабилизатора (при этом $l / R_{n}=$ $=0)$ ток $I$ протекает по ВТСП слою, $I_{s}=I$, и при учете линейного уменьшения критического тока $I_{c}(T)$ с ростом температуры из (4) следует, что

$$
\frac{\partial Q}{\partial T} \propto \frac{1}{\left(T_{c}-T\right)^{n+1}} .
$$

Вследствие большой величины показателя степени $n$ производная тепловыделения (5) резко возрастает при увеличении температуры, благодаря чему кривая тепловыделения $Q(T, I)$ может пересекать кривую теплоотвода $W(T)$ снизу или же вообще располагаться выше нее. В согласии с [3] это соответствует неустойчивому положению равновесия или вообще отсутствию такового.

При наличии стабилизирующего слоя его влияние на величину тепловыделения определяется значением полного тока в ВТСП ленте. Так, при полном токе $I$, равном критическому току $I_{c}\left(T_{0}\right)$, напряженность электрического поля, равная $E=E_{0}=10^{-4} \mathrm{~V} / \mathrm{m}$, вызывает в стабилизирующем слое протекание тока, на 3 порядка меньшего, чем ток в ВТСП слое (при типичных значениях толщины стабилизирующего слоя $-20 \mu \mathrm{m}$, его ширины $-12 \mathrm{~mm}$ и температуре $77 \mathrm{~K}$ ). Очевидно, что расчеты в этом случае покажут малое влияние стабилизатора на все процессы в ВТСП ленте, причем расчетная величина тока теплового срыва будет лишь незначительно превышать его значение в отсутствие стабилизатора. Именно этим случаем ограничено рассмотрение данного вопроса в [3].

Теперь рассмотрим стабилизированную ВТСП ленту при полном токе, значительно (приблизительно вдвое) превышающем критический ток. Тогда в силу степенного характера ВАХ ВТСП слоя и большого значения показателя $n$ напряженность поля $E$ возрастает на несколько порядков, в результате чего ток в стабилизирующем слое становится сравнимым по величине с током в ВТСП слое. В таком случае влияние стабилизатора весьма существенно, поэтому формула (5) неприменима, а для вычисления производной тепловыделения необходимо использовать выражение (4). Оценим значение (4) при температурах $T$, превышающих середину температурного интервала от $T_{0}$ до $T_{c}$ при полном токе ленты, большем величины критического тока при температуре хладагента $I>I_{c}\left(T_{0}\right)$. Следует учесть, что при бо́льших значениях $n$ анализируемая модель со степенной ВАХ дает результаты, близкие к модели критического состояния Бина [18]. А именно ток в сверхпроводящем слое достигает критического значения $I_{c}$ при $E=E_{0}$, после чего мало изменяется с ростом $E$. При этом по стабилизатору протекает ток $I_{n}=I-I_{s}$. При рассматриваемых температурах $T>\left(T_{0}+T_{c}\right) / 2$ из этого следует, что $I_{n}(T)>I_{s}(T)$. С учетом соотношения
$E(T)=I_{n}(T) R_{n} / l$ получается, что при $n \gg 1$ первое слагаемое в квадратных скобках выражения (4) мало по сравнению со вторым. В результате для производной тепловыделения при полных токах, превышающих критический ток $I_{c}\left(T_{0}\right)$, и при температурах от середины интервала $\left(T_{c}-T_{0}\right)$ до критической температуры $T_{c}$ производная (4) практически не зависит от температуры и приближенно равна

$$
\frac{\partial Q}{\partial T} \approx I \frac{I_{c}\left(T_{0}\right) R_{n}}{\left(T_{c}-T_{0}\right) l}
$$

при

$$
I>I_{c}\left(T_{0}\right),\left(T_{c}+T_{0}\right) / 2 \leq T \leq T_{c} .
$$

Таким образом, при токах, больших критического тока $I_{c}\left(T_{0}\right)$, и в широком интервале температур выше температуры хладагента $T_{0}$ зависимость тепловыделения ВТСП ленты со стабилизатором от температуры близка к прямо пропорциональной, а соответствующая ей кривая $Q(T, I)$ может пересекать более круто возрастающую кривую теплоотвода при кипении $W(T)$ сверху при определенном наборе параметров стабилизатора и в некотором диапазоне полных токов в композитной ВТСП ленте. Это означает, что наличие стабилизирующего покрытия может значительно (в разы) увеличить предельный ток ВТСП ленты, до которого существуют устойчивые резистивные состояния. Тепловой срыв, определяемый равенством тепловыделения и теплоотвода и равенством их производных (условия (3)), будет возможен только в области температур, где производная теплоотвода уменьшается. Это имеет место вблизи максимального значения теплоотвода при пузырьковом кипении, т.е. вблизи кризиса кипения. В этой области аналитического описания зависимости $W(T)$ не существует, а экспериментальные значения максимальной плотности теплового потока (первая критическая плотность теплового потока) находятся в диапазоне $(0.7-1.0) \cdot 10^{5} \mathrm{~W} / \mathrm{m}^{2}$ при пузырьковом кипении жидкого азота на металлических поверхностях толщиной в несколько десятых долей миллиметра [9]. Тем не менее, для качественной оценки тока срыва вблизи максимума кривой кипения достаточно использовать первое из условий (3), что будет сделано ниже.

Выводы из проведенного выше анализа подтверждаются результатами численного расчета, выполненного с использованием (1) и (2). В качестве параметров ВТСП ленты при расчетах принимались типичные для существующих лент значения: ширина ленты $-12 \mathrm{~mm}$, суммарная толщина стабилизатора медь/серебро - $20 \mu \mathrm{m}$ (толщина слоя серебра на порядок меньше, чем меди), периметр поперечного сечения $P \approx 24 \mathrm{~mm}$, критическая температура сверхпроводящего $\mathrm{YBaCuO}$ слоя ленты $92 \mathrm{~K}$, критический ток при температуре жидкого азота $I_{c}\left(T_{0}\right)=300 \mathrm{~A}$. Значения удельного сопротивления меди и серебра при температуре жидкого азота были взяты из [19].

На рис. 1 и 2 приведены рассчитанные зависимости тепловыделений $q$ с единицы длины ВТСП ленты, 


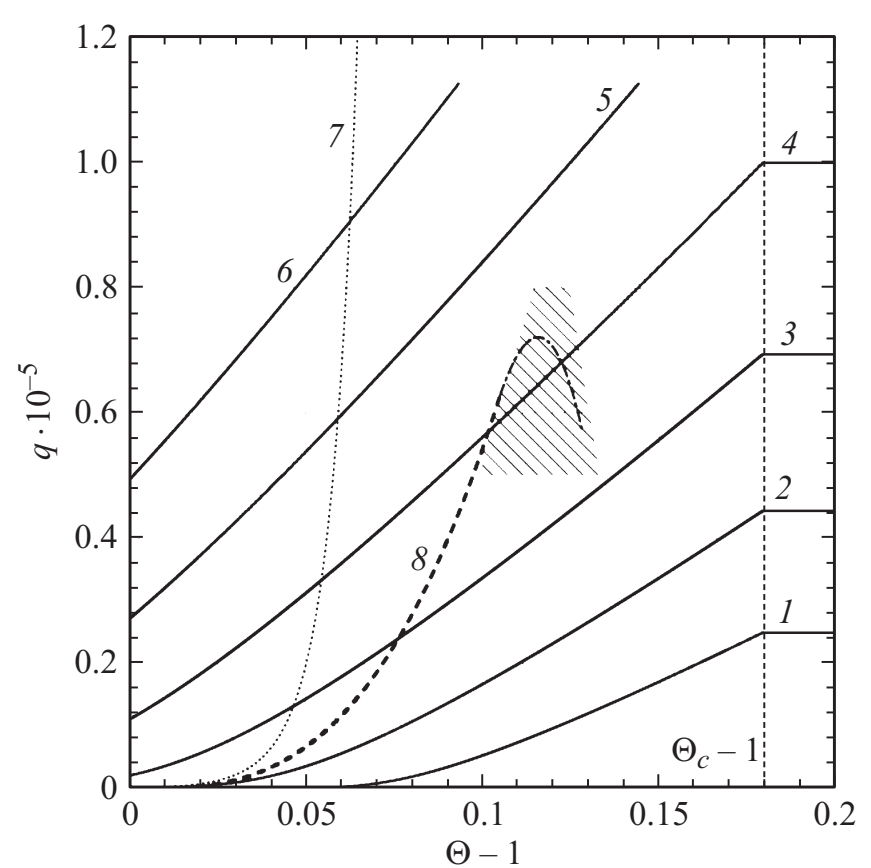

Рис. 1. Расчетные зависимости удельного тепловыделения от температуры в ВТСП ленте $(n=15)$ со стабилизирующим покрытием (кривые 1-6) и без него (кривая 7) и теплового потока в жидкий азот (кривая 8). Значения $i: 1-1.00,2-$ $1.33,3-1.67,4-2.00,5-2.33,6-2.67,7-1.37$.

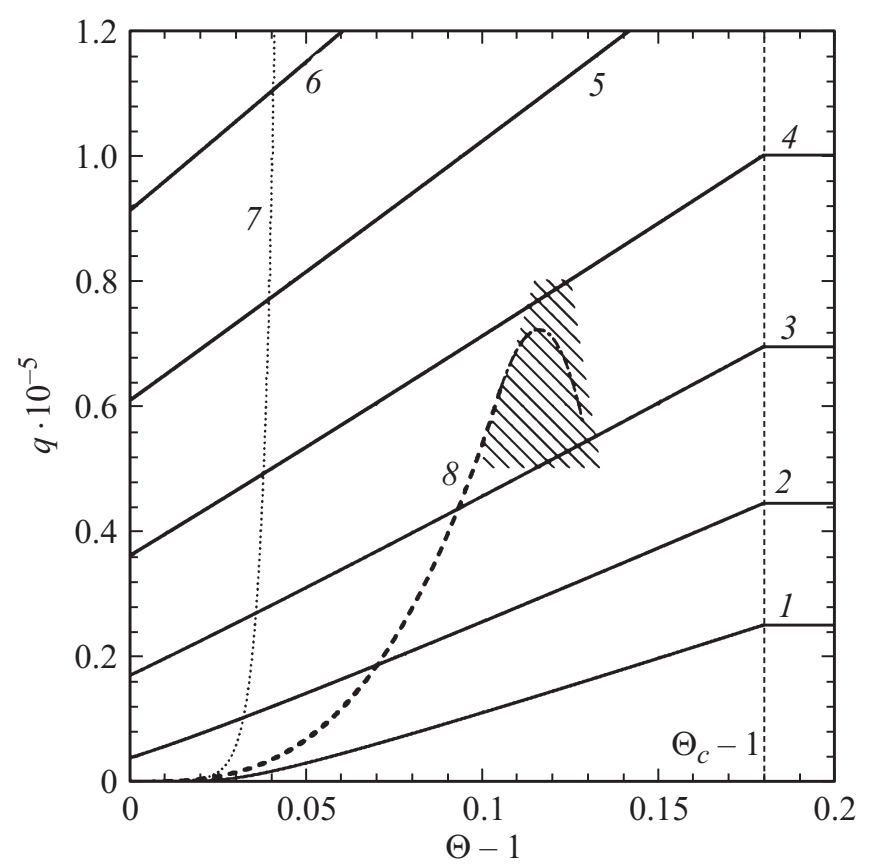

Рис. 2. Расчетные зависимости удельного тепловыделения от температуры в ВТСП ленте $(n=40)$ со стабилизирующим покрытием (кривые $1-6$ ) и без него (кривая 7) и теплового потока в жидкий азот (кривая 8). Значения $i: 1-1.00,2-$ $1.33,3-1.67,4-2.00,5-2.33,6-2.67,7-1.04$.

выраженных в безразмерном виде $q=Q /\left(E_{0} I_{c}\left(T_{0}\right)\right)$, от безразмерной температуры $\Theta=T / T_{0}$. Для показателей степени ВАХ в реальных ВТСП лентах наиболее часто встречаются значения от $n=15$ [8] до $n=40$ [11], поэтому на рис. 1 и 2 приведены результаты расчетов для граничных значений $n$ из этого диапазона. Кривые $1-6$ на обоих рисунках соответствуют увеличению тока в ленте от 300 А до 800 А с шагом $100 \mathrm{~A}\left(i=I / I_{c}\left(T_{0}\right)-\right.$ приведенный ток) при наличии в лентах стабилизатора с электропроводностью, равной электропроводности слоя медь/серебро при температуре $77 \mathrm{~K}$, кривые 7 - в его отсутствие.

Кривая 8 представляет собой выраженную в безразмерном виде расчетную зависимость теплового потока в хладагент $w$ с единицы длины ленты при пузырьковом кипении жидкого азота, $w=W(T) /\left(E_{0} I_{c}\left(T_{0}\right)\right)=$ $=q_{N 2}(\Delta T) P /\left(E_{0} I_{c}\left(T_{0}\right)\right)$. Для получения зависимости плотности теплового потока от теплового напора $q_{N 2}(\Delta T)$ использовалось уравнение для расчета коэффициента теплоотдачи $\alpha$ при пузырьковом кипении $\alpha \propto q_{N 2}^{2 / 3}$ (формулы (3-18) в [9]) и соотношение $q_{N 2}=\alpha \Delta T$. В результате для плотности теплового потока получается выражение

$$
q_{N 2}=q_{0}(\Delta T)^{3}
$$

где $q_{0}-$ постоянная, численное значение которой, рассчитанное с использованием значений параметров хладагента (теплопроводность, плотность, вязкость, коэффициент поверхностного натяжения) [19], для жидкого азота равно $q_{0} \sim 1.4 \cdot 10^{2} \mathrm{~W} /\left(\mathrm{m}^{2} \mathrm{~K}^{3}\right)$. В обезразмеренном виде уравнение кривой 8 , выраженное через безразмерную температуру $\Theta$, имеет вид $w(\Theta)=w_{0} \cdot(\Theta-1)^{3}$, где $w_{0} \approx 5.4 \cdot 10^{7}$.

Заштрихованная область соответствует разбросу экспериментальных данных в области кризиса кипения азота [9], а численные значения ее границ представлены, как и все кривые на рис. 1 и 2, в безразмерном виде. Размерные значения получаются посредством умножения на $E_{0} \cdot I_{c}\left(T_{0}\right) / P \approx 1.25 \mathrm{~W} / \mathrm{m}^{2}$ и соответствуют указанному выше диапазону первой критической плотности теплового потока.

В ленте без слоя стабилизатора (рис. 1 и 2, кривые 7) отсутствие положения теплового равновесия имеет место уже при небольшом превышении током критического значения, причем с ростом показателя степени $n$ модельной ВАХ это превышение сильно снижается.

При наличии стабилизатора устойчивое положение равновесия существует вплоть до прохождения кривой тепловыделения через область кризиса кипения. Срыв устойчивого состояния будет происходить при значениях тока, соответствующих кривым тепловыделения, расположенным вблизи кривой 4 на рис. 1 и 2. Это означает, что срыв происходит при значениях тока в ВТСП ленте, почти в 2 раза превышающих критический ток при температуре хладагента, т.е. при $I / I_{c}\left(T_{0}\right) \approx 2$ (конкретное значение предельного тока устойчивого равновесия определяется показателем степени $n$ в модельной ВАХ). Этот результат отличен от полученного 
в [3], где рассмотрен случай стабилизирующего покрытия, которое не оказывает заметного влияния на ток перехода. Таким образом, наличие стабилизирующего слоя с высокой электропроводностью для повышения тепловой стабильности имеет для ВТСП лент не менее существенное значение, чем для низкотемпературных композитных сверхпроводников.

\section{2. Экспериментальное исследование ВТСП лент в перегруженном режиме}

Качественный анализ влияния стабилизирующего слоя в ВТСП лентах был выполнен в разд. 1 для постоянного тока, а разработанная в [4] теория для переменного тока не учитывает наличие кризиса кипения. Поэтому возникла необходимость провести экспериментальную проверку предположения о существенной роли стабилизатора в ВТСП лентах второго поколения в условиях, при которых они используются в реальном электрооборудовании, т.е. на переменном токе промышленной частоты и при охлаждении жидким азотом [17].

Для экспериментального исследования электрических и тепловых процессов в композитных ВТСП лентах в режиме токовой перегрузки была применена описанная в [1] методика измерений на переменном токе частотой $50 \mathrm{~Hz}$.

Изучались ВТСП материалы компании SuperPower Inc. [20] трех типов: SF12100 (SF - структура без слоя стабилизатора, 12 - ширина ленты в mm, 100 - толщина слоя подложки (сплав Hastelloy (На) в $\mu \mathrm{m}$, толщина защитного слоя серебра - $1.5 \mu \mathrm{m}), \operatorname{SF} 12050$ (50 толщина слоя подложки в $\mu \mathrm{m}$, толщина защитного слоя серебра - $2 \mu \mathrm{m}), \mathrm{SCS} 12050$ (SCS - структура с медным стабилизатором с толщиной $10 \mu \mathrm{m}$ с каждой стороны ленты, 12 - ширина ленты в $\mathrm{mm}, 50$ - толщина слоя подложки в $\mu \mathrm{m}$, толщина защитного слоя серебра $2 \mu \mathrm{m})$.

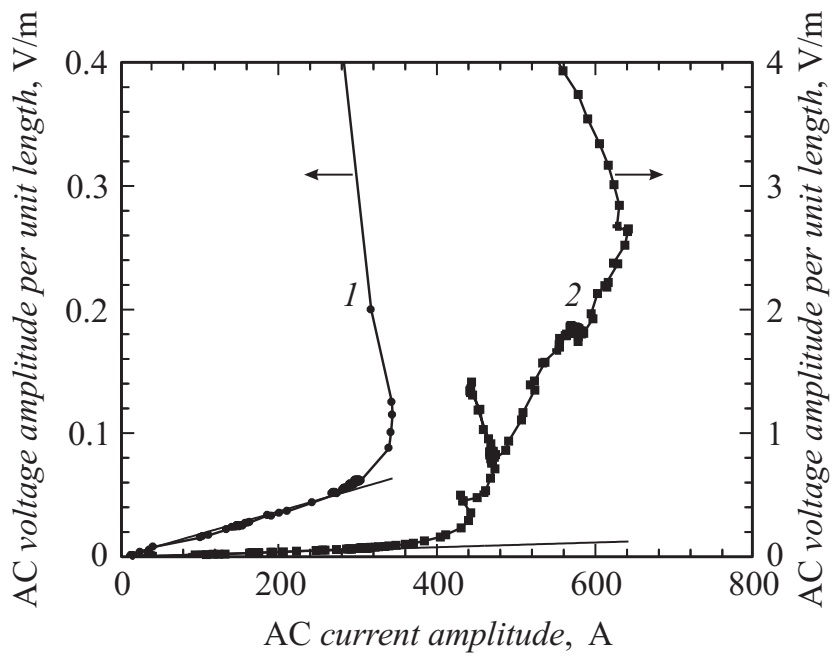

Рис. 3. Амплитудные BAX на переменном токе: 1 - образец SF12050, 2 - образец SCS12050.
Результаты измерений амплитудных ВАХ [16] на переменном токе для образцов ВТСП лент приведены на рис. 3 для SF12050 (кривая 1) и SCS12050 (кривая 2) лент. По оси ординат отложено среднее по длине образца ленты амплитудное значение напряженности электрического поля $E$, которое до точки теплового срыва распределено вдоль ленты равномерно (см. разд. 1). Обе ВАХ имеют начальный линейный участок, обусловленный индуктивным сопротивлением ленты в сверхпроводящем состоянии.

При превышении амплитудой тока критического значения происходит возрастание крутизны ВАХ вследствие возникновения резистивной составляющей полного сопротивления образца. При амплитуде тока $I_{q}=340$ А для ленты без медного слоя и $I_{q}=640$ А для ленты с медным стабилизирующим слоем начинается переходный процесс, сопровождающийся уменьшением тока и повышением напряжения. В результате происходит тепловой переход в область отсутствия равновесного состояния и сильного разогрева ленты. Следовательно, наличие стабилизирующего покрытия толщиной $20 \mu \mathrm{m}$ приводит к повышению тока теплового перехода в 1.9 раза по сравнению с лентой, где покрытие отсутствует.

Оценку тепловыделений при токе, равном току перехода, можно сделать на основании расчетных результатов работы [4], где показано, что при частоте переменного тока в несколько десятков герц амплитудные значения тока и напряжения в ВТСП в перегруженном режиме достигаются практически одновременно, причем переход в неустойчивое тепловое состояние определяется мгновенным значением тепловыделения. При значениях токов и напряженностей электрического поля в точках теплового перехода, определенных по рис. 3, получаем для плотности теплового потока $0.7 \cdot 10^{5} \mathrm{~W} / \mathrm{m}^{2}$ для стабилизированной ленты и на два порядка меньшее значение для ленты без слоя меди. Такие величины тепловых потоков согласуются с проведенным в разд. 1 анализом причины теплового перехода: попадание в область кризиса кипения (для тонких металлических слоев) в случае стабилизированных ВТСП лент или достижение границы между устойчивыми и неустойчивыми состояниями для ВТСП ленты без стабилизатора [3].

Следует заметить, что на кривой 2 (рис. 3) при токе около 470 А имеется ответвление, соответствующее переходному процессу, который заканчивается возвращением на основную ветвь ВАХ. Подобный переходный процесс может являться следствием задержки активации пузырькового кипения при смене механизма теплоотвода в жидкий азот от конвекции к пузырьковому кипению [7].

\section{3. Исследование тепловых процессов при переходе ВТСП ленты В нормальное состояние}

Рассмотрим процесс охлаждения ВТСП ленты, разогретой до температуры $T$ посредством большой токовой перегрузки и затем возвращающейся в состояние 


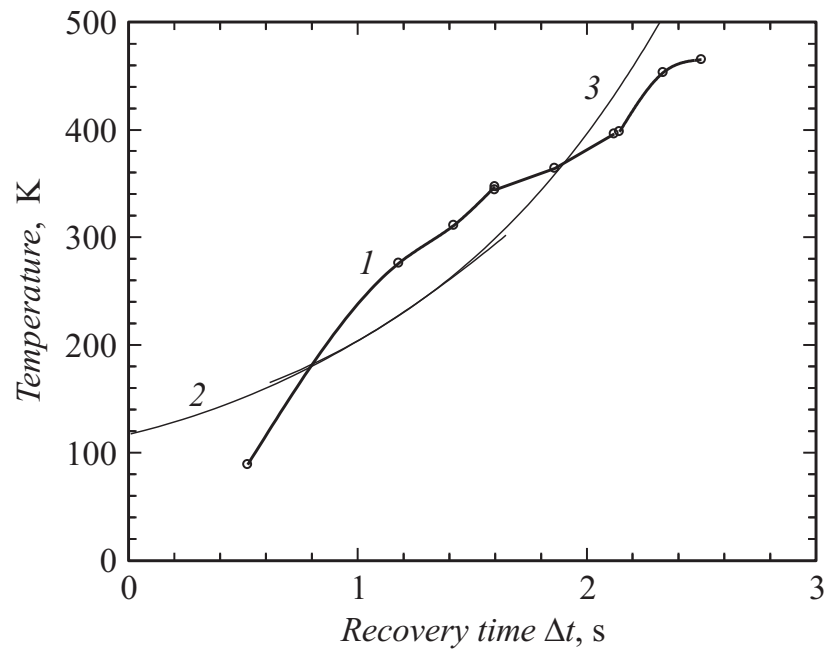

Рис. 4. Взаимозависимость температуры и времени восстановления сверхпроводимости для SF12100: 1 - эксперимент, 2 и 3 - выражение (12).

теплового равновесия с хладагентом после отключения тока. В этом случае нормальная зона сосредоточена в небольшой области нормального домена [1], и уравнение теплового баланса в этой области выглядит следующим образом:

$$
C(T) s d T=\operatorname{abg}(T) d t
$$

В уравнении (8) $C(T)$ - объемная теплоемкость ленты (для ВТСП ленты теплоемкости металлов, входящих в ее состав, близки, поэтому можно считать что $C(T)$ определяется теплоемкостью меди), $s$ - толщина ленты, $d T$ - малое изменение средней температуры домена, $d t$ - малый промежуток времени в процессе охлаждения, $g(T)$ - плотность теплового потока в хладагент, $a$ и $b-$ безразмерные параметры, учитывающие особенности продольного и поперечного тепловых потоков внутри ВТСП ленты. Введение этих параметров и их количественная оценка позволит наиболее детально представить характер ее разогрева. Параметр $a$ связан с эффективным увеличением размера домена (когда теплоотвод в хладагент идет только от разогретой центральной части домена $a=1$, когда есть дополнительный теплоотвод от фронтальных областей домена $a>1$ ). Параметр $b$ учитывает вклад каждой из поверхностей ленты в суммарный теплоотвод (когда теплоотводы с поверхности ВТСП слоя и от подложки (На) равны, то $b=2$, а когда тепловой поток со стороны На отсутствует, то $b=1$, то есть $1<b<2$ ). В первом приближении $a=1$ и $b=2$. Для их количественного уточнения проведем цикл вычислений с привлечением экспериментальных данных. На начальной стадии для $a=1$ и $b=2$ из соотношения (8) получаем в интегральном виде выражение для времени восстановления сверхпроводящего состояния $\Delta t$ при остывании образца от температуры $T$ до температуры азотной ванны в $77 \mathrm{~K}$ :

$$
\Delta t=\int_{77}^{T} \frac{s C(T)}{2 g(T)} d T \approx \int_{117}^{T} \frac{s C(T)}{2 g_{f}(T)} d T .
$$

В (9) принято во внимание, что при пузырьковом и переходном кипении при температурах от 77 до $117 \mathrm{~K}$ вклад в интегральное выражение (9) мал, поскольку параметр $g(T)$ в этом диапазоне на порядок выше, чем плотность теплового потока при пленочном кипении $g_{f}(T)$; при этом наибольший интерес представляют только такие условия нагрева при токовой перегрузке, при которых $T$ намного больше, чем $117 \mathrm{~K}$.

При проведении оценочных расчетов используем справочные данные для материалов, из которых состоит ВТСП лента, в том числе температурные зависимости удельных сопротивлений сплава Hastelloy [20], меди, серебра и удельной объемной теплоемкости меди [19]:

$$
\begin{aligned}
& C(T)= \\
& \left\{\begin{array} { l } 
{ a _ { 2 } + b _ { 2 } ( T - 7 7 ) = 2 . 1 2 + 0 . 0 0 8 7 ( T - 7 7 ) , } \\
{ 1 1 7 \mathrm { K } \leq T \leq 2 1 2 \mathrm { K } , } \\
{ a _ { 3 } + b _ { 3 } ( T - 7 7 ) = 3 . 2 6 5 + 0 . 0 0 1 ( T - 7 7 ) , } \\
{ 2 1 2 \mathrm { K } \leq T \leq 8 0 0 \mathrm { K } . }
\end{array} \left[\mathrm{J} /\left(\mathrm{cm}^{3} \mathrm{~K}\right]\right.\right.
\end{aligned}
$$

Плотность теплового потока с поверхности ВТСП ленты в жидкий азот в режиме пленочного кипения задается следующим выражением [10]:

$$
\begin{gathered}
g_{f}(T)=b_{1}(T-77)=0.016(T-77) \quad\left[\mathrm{W} / \mathrm{cm}^{2}\right], \\
40 \mathrm{~K} \leq T-77 \leq 800 \mathrm{~K} .
\end{gathered}
$$

Для упрощения расчетов температура отсчитывается от температуры кипения жидкого азота $T_{0}=77 \mathrm{~K}$, при которой до начала нагрева током находятся ВТСП образцы.

Подставляя в выражение (9) значения для $C(T)$ и $g_{f}(T)$ в явном виде (соотношения (10) и $\left.(11)\right)$, получаем зависимость $\Delta t$ от температуры

$$
\Delta t=\left\{\begin{array}{l}
\left.\frac{s a_{2}}{2 b_{1}} \ln (T-77)\right|_{117} ^{T}+\left.\frac{s b_{2}}{2 b_{1}}(T-77)\right|_{117} ^{T}, \\
117 \mathrm{~K} \leq T \leq 212 \mathrm{~K}, \\
\left.\frac{s a_{3}}{2 b_{1}} \ln (T-77)\right|_{212} ^{T}+\left.\frac{s b_{3}}{2 b_{1}}(T-77)\right|_{212} ^{T}+\Delta t(212 \mathrm{~K}), \\
212 \mathrm{~K} \leq T \leq 800 \mathrm{~K} .
\end{array}\right.
$$

Выражения (12) устанавливают связь между температурой, до которой разогрелась нормальная область во время протекания тока, и временем восстановления сверхпроводимости.

Экспериментальная проверка (12) проводилась следующим образом. На образец подавались прямоугольные импульсы переменного напряжения частотой $50 \mathrm{~Hz}$ 
различной амплитуды длительностью 5-7 s. Синхронно с этим напряжением измерялась температура нормальной области. Время восстановления сверхпроводимости определялось как по спаду температуры, так и по спаду сопротивления после скачкообразного снижения напряжения. Полученная таким образом взаимозависимость этого времени и измеряемой температуры приведена на рис. 4. Как было показано в работе [1], при малых напряжениях нормальная область удалена от термопары, и термопара реагирует только на периферию разогретой области (первая слева экспериментальная точка). По мере увеличения размера нормального домена термопара оказывается в его центре и показывает его максимальную температуру (экспериментальные точки вблизи $300 \mathrm{~K}$ ). Дальнейшее повышение напряжения приводит к неустойчивости домена и его смещению вдоль ленты. В этом случае термопара регистрирует температуру вблизи фронта домена. Таким образом, расчетные кривые, описываемые выражением (12), отражают среднюю температуру разогретой области. Экспериментальная зависимость времени восстановления от амплитуды напряжения $V_{0}$ для трех образцов с различной структурой представлена на рис. 5. Функциональная зависимость $\Delta t$ от $V_{0}$ в каждом из трех характерных по $V_{0}$ диапазонах $\left(0 \mathrm{~V} \leq V_{0} \leq 0.5 \mathrm{~V}, 0.5 \mathrm{~V} \leq V_{0} \leq 0.8 \mathrm{~V}\right.$, $\left.0.8 \mathrm{~V} \leq V_{0} \leq 2.0 \mathrm{~V}\right)$ для всех образцов аппроксимируется выражением следующего вида:

$$
\Delta t=A V_{0}^{\lambda}
$$

Заменив малые по величине члены линейной температурной зависимости в (12) в каждом температурном диапазоне на усредненное постоянное значение в этом диапазоне, используя $\Delta t$ как параметр и сравнивая выражение (12) с измеренными значениями $\Delta t(13)$, найдем первое приближение для актуальной при практических применениях ВТСП лент взаимозависимости температу-

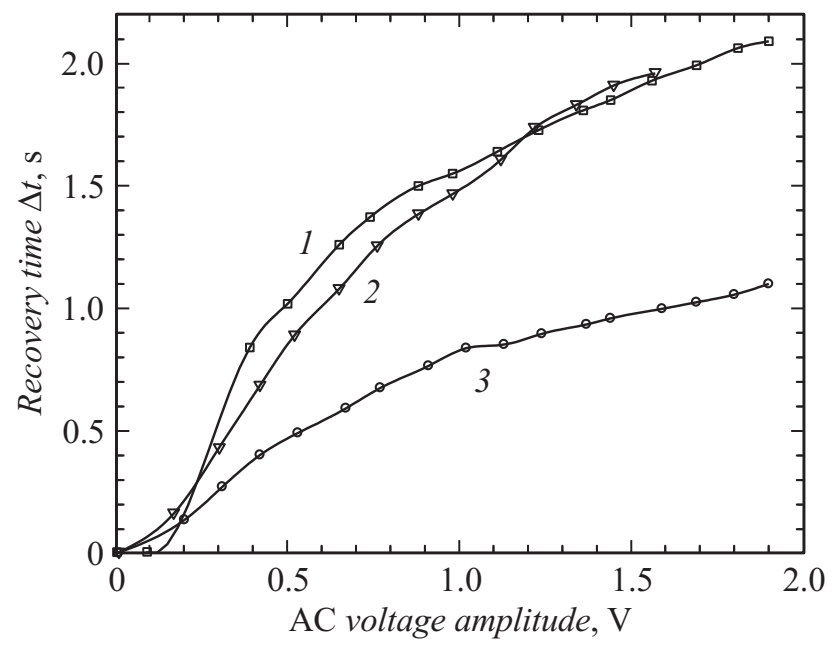

Рис. 5. Зависимости времени восстановления сверхпроводимости от амплитуды напряжения. 1 - образец SCS12050, 2 SF12100, 3 - SF12050.

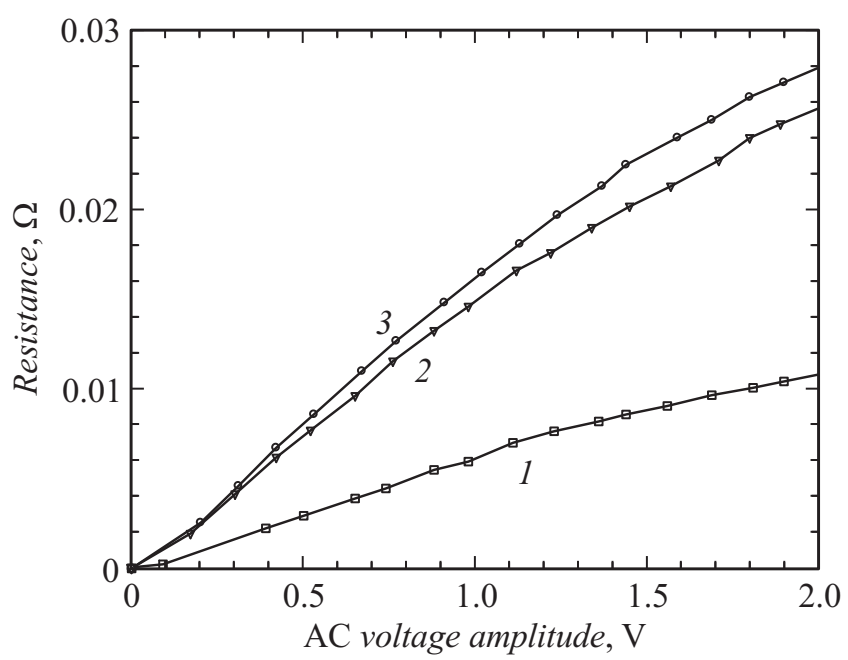

Рис. 6. Зависимости сопротивления от амплитуды напряжения $V_{0}: 1$ - образец SCS12050, 2 - SF12100, 3 - SF12050.

ры и амплитуды приложенного напряжения:

$$
\begin{gathered}
T=77+A_{1} \exp \left(A_{2} V_{0}^{\lambda}\right), \\
V_{0}=\left(A_{2}^{-1} \ln (T-77)-A_{3}\right)^{1 / \lambda},
\end{gathered}
$$

где $A_{1}, A_{2}, A_{3}$ - численные коэффициенты, которые являются комбинациями эмпирических коэффициентов из вышеприведенных справочных данных по теплоемкости ленты (10) и характеру теплоотвода (11), параметров образцов и экспериментальных данных (13).

Выражения (8)-(15) получены при анализе процесса остывания ВТСП лент в случае отсутствия джоулевых тепловыделений. Независимый набор данных, позволяющий найти присутствующие в (8) параметры теплоотвода в явном виде, можно получить из анализа процесса разогрева ленты во время ее перехода в нормальное состояние при наличии тепловыделений. В этом случае уравнение теплового баланса выглядит следующим образом:

$$
\frac{V_{0}^{2} s h}{2 r(T) D}=\operatorname{abg}(T) h D .
$$

В выражении (16) слева записана мощность тепловыделений в образце ( $V_{0}-$ амплитуда напряжения), а справа — теплоотвод в единицу времени, $r(T)-$ эквивалентное удельное сопротивление образца, $g(T)-$ плотность теплового потока в хладагент, $D-$ размер домена, $h$ и $s$ - высота и толщина образца, $a$ и $b-$ параметры теплоотвода. Из выражения (16) можно найти выражение для размера домена и его сопротивления $R$.

$$
\begin{gathered}
D=V_{0} \sqrt{\frac{s}{2 a b r(T) g(T)}}, \\
R=\frac{V_{0}}{h} \sqrt{\frac{r(T)}{2 a b s g(T)}} .
\end{gathered}
$$




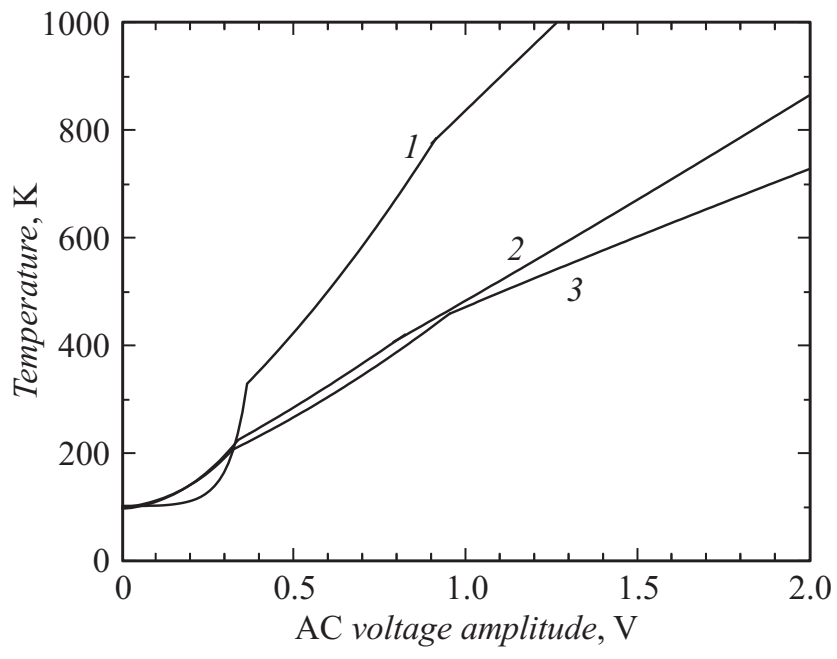

Рис. 7. Зависимости температуры от напряжения: 1 - образец SCS12050, 2 - SF12100, 3 - SF12050.

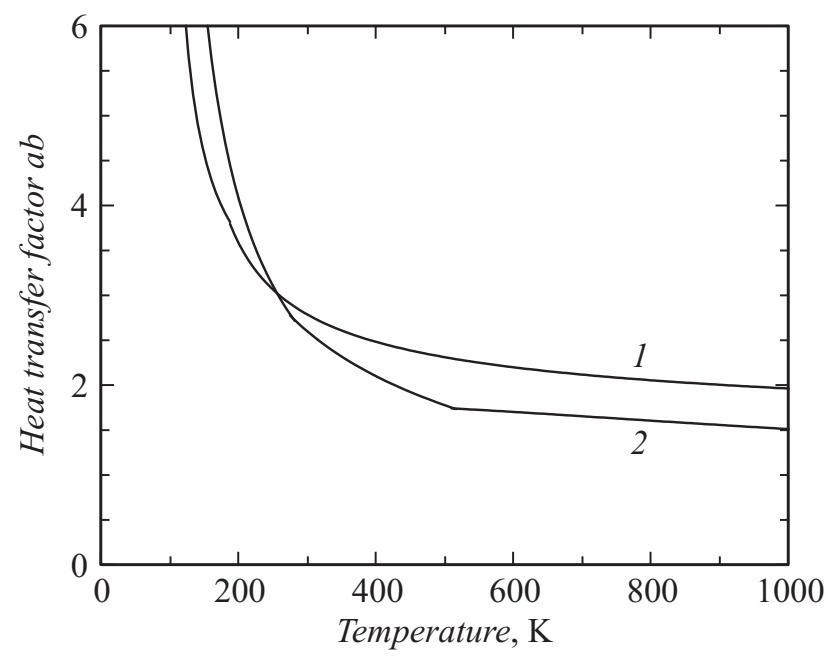

Рис. 8. Зависимости произведения параметров теплоотвода от температуры: 1 - образец SCS12050, 2 - SF12100.

Полученные в экспериментах значения $R$ для исследованных образцов представлены на рис. 6. Зависимости $R$ от $V_{0}$ для всех образцов аппроксимируются степенной функцией, где значения показателя степени $\beta$ определяются диапазоном изменения амплитуды напряжения:

$$
R=B V_{0}^{\beta}
$$

Приравнивая аппроксимационную формулу для $R$ к выражению (18), получаем зависимость произведения параметров теплоотвода $a b$ от напряжения и температуры:

$$
a b=V_{0}^{2(1-\beta)} \frac{r(T)}{2 B^{2} h^{2} s g(T)} .
$$

Подставляя численные значения параметров и явный вид $r(T)$, а также зависимость $V_{0}(T)$ (выражение (15), полученное из анализа процесса охлаждения ВТСП ленты), находим в первом приближении температурную зависимость для произведения параметров теплоотвода. Подставляя эту зависимость в уравнение (8) и используя экспериментальные данные (13), получаем уточненную взаимосвязь $T$ и $V_{0}$. Для ВТСП лент с различной структурой зависимости температуры домена от приложенного напряжения приведены на рис. 7.

Уточненный вид зависимости произведения параметров теплоотвода от температуры получаем с помощью уравнения (20), экспериментальных данных (19) и уточненной взаимосвязи напряжения и температуры.

На рис. 8 приведены температурные зависимости произведения параметров теплоотвода. Видно, что для образцов SF12100 (без медной оболочки) и SCS12050 (с наличием медного стабилизатора) существует принципиальное различие в поведении указанных параметров. В образце SCS12050 после перехода его в нормальное состояние основные тепловыделения происходят в медном стабилизаторе, который расположен равномерно с обеих сторон ленты, теплоотвод с двух сторон ленты одинаков, а параметр теплоотвода $b=2$. Таким образом, поделенные на $b=2$ значения произведения параметров, приведенные на кривой 1 , выражают параметр теплоотвода $a$, который характеризует увеличение эффективного размера домена в образце SCS12050 за счет продольного отвода тепла. Как следует из рис. $8, a(T)-$ это убывающая функция температуры, которая при больших значениях $T$ стремится к единице. Кривая $2-$ это произведение параметров $a$ и $b$ для образца SF12100. Зная пределы изменения и их общий характер поведения, можно получить качественные характеристики этих параметров. Поскольку при $T>400 \mathrm{~K}$ произведение $a b<2$ (рис. 8), а параметр $a \geq 1$, то параметр $b<2$ и уменьшается по мере роста $T$. А это означает, что даже для квазистационарного разогрева теплоотвод со стороны подложки (На) значительно ниже, чем со стороны ВТСП слоя. Поскольку температура линейно связана с теплоотводом (выражение (11)), то в этой ситуации существует и значительный поперечный температурный градиент.

\section{Заключение}

В настоящей работе исследованы тепловые процессы на разных стадиях перехода из сверхпроводящего в нормальное состояние в многослойных ВТСП проводниках при токовой перегрузке.

Установлено, что при наличии стабилизирующего слоя значение тока теплового перехода ВТСП ленты из резистивного в нормальное состояние повышается более чем в 2 раза по сравнению с соответствующим током для нестабилизированной ленты.

Для ВТСП лент посредством применения расчетной модели определена взаимозависимость между температурой разогрева и временем возврата из нормальной 
в сверхпроводящую фазу после отключения приложенного напряжения. Установленная закономерность и экспериментальные данные по времени восстановления сверхпроводящего состояния позволили получить важную для применений в электротехнических устройствах зависимость температуры разогрева от приложенного напряжения.

Обнаружено, что у ВТСП материалов без низкоомного стабилизирующего слоя за счет теплового барьера на буферном слое во время их перехода в нормальное состояние возникает значительный поперечный температурный градиент, что приводит к существенному отличию теплоотвода с разных сторон ленты. Учет этого явления и экспериментальные данные по сопротивлению нормальной зоны позволяют значительно уточнить оценку максимального разогрева ВТСП ленты в нормальном состоянии.

Авторы благодарят А.Ю. Кунцевича за помощь в проведении экспериментов и за критические замечания при обсуждении их результатов. Особую благодарность авторы выражают В.М. Пудалову за постоянную поддержку выбранного направления изучения ВТСП материалов и за ряд замечаний при подготовке данной публикации.

Работа выполнена на оборудовании ЦКП ФИАН, при финансовой поддержке РНФ в рамках научного проекта № 16-42-01100.

\section{Список литературы}

[1] Мальгинов А.В., Кунцевич А.Ю., Мальгинов В.А., Флейшман Л.С. // ЖЭТФ. 2013. Т. 144. Вып. 6. С. 1225-1238.

[2] Гуревич А.Вл., Мини, Р.Г., Рахманов А.А. Физика композитных сверхпроводников. М.: Наука, 1987. С. 9.

[3] Рахманов А.Л., Иванов С.С. // Электричество. 2015. № 10. C. $38-47$.

[4] Романовский В.Р. // ЖТФ. 2015. Т. 85. Вып. 1. С. 87-97.

[5] Романовский В.Р. // ЖТФ. 2017. Т. 87. Вып. 4. С. 540-548.

[6] Fetisov S.S., Vysotsky V.S., Sytnikov V.E. // IEEE Trans. Appl. Supercond. 2009. Vol. 19, N 3. P. 2411-2414.

[7] Fetisov S.S., Vysotsky V.S., Zubco V.V. // IEEE Trans. Appl. Supercond. 2011. Vol. 21. N 3. P. 1323-1327.

[8] Zubko V.V., Ryabov S.M., Fetisov S.S. et al. // Phys. Proc. 2015. Vol. 67. P. 619-624.

[9] Григорьев В.А., Павлов Ю.М., Аметистов Е.В. Кипение криогенных жидкостей. М.: Энергия, 1977. 288 с.

[10] Roy F. 2nd International Workshop on Modeling HTS. Cambridge, UK, 2011. P. 18.

[11] Choi J.H., Choi Y.H., Kang D.-H. et al. // IEEE Trans. Appl. Supercond. 2015. Vol. 25. N 3. P. 6600505.

[12] Флейшман Л.С. // Электрические станции. 2005. № 8. C. $71-75$.

[13] Флейшман Л.С., Мальгинов В.А., Мальгинов А.В. В сб.: Тез. докл. IV Межд. конференции ФПС'11. М.: ФИАН. (2011). C. 291.

[14] Волков Э.П., Флейшман Л.С., Мальгинов В.А. и др. // Известия РАН. Энергетика. 2009. № 2. С. 64-76.

[15] Флейшман Л.С., Мальгинов В.А., Мальгинов А.В. // Известия РАН. Энергетика. 2010. № 5. С. 61-67.
[16] Fleishman L.S., Volkov E.P., Malginov V.A. et al. // IEEE Trans. Appl. Supercond. 2011. Vol. 21. N 3. P. 1263-1266.

[17] Волков Э.П., Джсафаров Э.А., Флейшман Л.С., Высоикий В.С., Суконкин В.В. // Известия РАН. Энергетика. 2016. № 5. C. 45-56.

[18] Carr Jr. W.J. AC Loss and Macroscopic Theory of Superconductors. L.: Taylor \& Francis, 2001. P. 172.

[19] Справочник по физико-техническим основам криогеники / Под ред. М.П. Малкова. М.: Энергоатомиздат, 1985. 432 с.

[20] SuperPower 2G HTS Wire. http://www.superpower-inc.com/content/wire-specification 